\title{
Recursos para Flipped Learning: Moodle versus Edpuzzle
}

\author{
Nuria E. Gallardo López ${ }^{a}$, Antonia M. Caleya Zambranob, Gonzalo Feijoo García ${ }^{\mathrm{c}}$, \\ Esperanza Sánchez Sánchez ${ }^{\mathrm{d}}$, Joaquín de Nova García ${ }^{\mathrm{e}}$, Rosa Mourelle Martínez ${ }^{\mathrm{f}}$ \\ Departamento Especialidades Clínicas Odontológicas. Facultad de Odontología. Universidad \\ Complutense de Madrid. España. \\ anegallar@ucm.es, bamcaleya@ucm.es, 'cffeijoo@ucm.es, ${ }^{\mathrm{d}}$ maresanc@ucm.es, ddenova@ucm.es, \\ f mrmourel@ucm.es
}

\section{Resumen}

La principal particularidad del modelo invertido de aprendizaje o flipped learning (FL) radica en el cambio de roles profesor-estudiante y lo que supone respecto a la distribución del tiempo para ambos. Durante la clase, no se incorporan nuevos conocimientos, estos los adquiere el alumno con anterioridad, pero se resuelven dudas y se realizan prácticas o ejercicios con el apoyo del profesor.

Para los docentes, esta inversión del uso del tiempo marca una gran diferencia entre los modelos de aprendizaje invertido y tradicional. Utilizar FL es complejo y requiere tiempo. Se deben establecer objetivos de aprendizaje, planificar la secuencia de actividades, crear materiales de alta calidad y dar acceso a los estudiantes al contenido antes y fuera de la clase.

El objetivo de este trabajo fue comparar el uso de 2 recursos digitales (Moodle y Edpuzzle) en las prácticas de Odontopediatría del Grado de Odontología de la Universidad Complutense de Madrid.

Solo los alumnos matriculados en la asignatura participaron en el estudio, divididos en 3 grupos de 25 alumnos, con 2 profesores encargados de cada uno. Se aplicó FL en 2 grupos que utilizaron distintos recursos (A: Moodle y B: Edpuzzle) mientras que los alumnos restantes (grupo C) recibieron docencia presencial tradicional. Todos los alumnos tuvieron acceso a los vídeos demostrativos del procedimiento objeto de las prácticas.

Nuestros resultados demostraron que podemos realizar FL con distintos recursos digitales, como Moodle y Edpuzzle. Sin embargo, las calificaciones obtenidas por los alumnos fueron superiores con Edpuzzle y por tanto, su aprendizaje mejoró al compararlo con Moodle y con la enseñanza tradicional. Concluimos que resulta más adecuado el uso de Edpuzzle para la aplicación de FL en las prácticas de Odontopediatría.

Palabras clave: Flipped Learning, Aula invertida, Moodle, Edpuzzle 


\section{Introducción}

El modelo invertido de aprendizaje o flipped learning (FL) está basado en invertir la estructura tradicional de la clase presencial expositiva a través del empleo de tecnologías de información y comunicación (Bergmann y Sams, 2012). Se podría decir que hay un cambio de roles entre profesor y estudiante y también, una modificación estructural en cuanto a la distribución del tiempo empleado para la preparación y realización de las clases para ambos. Así, la adquisición de nuevos conocimientos por parte de los alumnos comienza con anterioridad a las clases presenciales, es decir, se produce usando las herramientas que el profesor les proporciona y que, generalmente, requieren el apoyo de plataformas educativas.

La taxonomía de Bloom estructuró en niveles la adquisición del conocimiento que, en cierta forma, se invierten al realizar FL. Es decir, la información básica del contenido, como recordar o entender, llega individualmente al alumno y le ayuda a trabajar su creatividad. Pero los aprendizajes más complejos desde el punto de vista cognitivo, son grupales, presenciales y son dirigidos por el docente (Campión, 2019).

Empezar a utilizar FL es complejo ya que la elaboración del material que se debe proporcionar al estudiante requiere por un lado, el aprendizaje de tecnologías informáticas, por ejemplo para crear vídeos, y mayor tiempo que las clases magistrales con las que usualmente se trabaja. Sin embargo, no es menos cierto, que una vez elaborado el material, éste puede ser mucho más versátil y facilitar el resto de las fases educativas, como la evaluación. Además, en la clase presencial la participación del docente disminuye sustancialmente, ya que se limita a la resolución de dudas y a realizar apoyo en las prácticas o ejercicios que el alumno debe resolver.

Podríamos entonces decir que para el desarrollo de FL es necesario establecer previamente: los objetivos de aprendizaje, la planificación de la secuencia de actividades, la creación de materiales de alta calidad y las plataformas educativas de apoyo que faciliten a los estudiantes acceso al contenido.

Existen en la actualidad múltiples plataformas educativas creadas especialmente para el desarrollo de FL, tales como, Edpuzzle, Panopto, eduCanon, Google Forms, Socrative, etc... Tras analizar varias, elegimos Edpuzzle para la realización de este estudio, por su accesibilidad, facilidad de uso y por permitir la evaluación y análisis de las clases. Todo ello hace que sea considerada una herramienta muy completa para ser utilizada en FL.

Edpuzzle es una aplicación gratuita que destaca por su versatilidad en la creación de contenidos audiovisuales (vídeos), que el alumnado puede ver desde dispositivos digitales como ordenadores, tablets o smartphones (Singh, Abdellahi, Maher, \& Latulipe, 2016). La 
ventaja más importante de Edpuzzle es que no solo se puede trabajar con vídeos de fabricación propia sino que permite personalizar vídeos ya preparados por otros (incluidos repositorios como You Tube), lo que facilita enormemente la elaboración del material para la FL. Al crear la clase, se puede editar el vídeo y realizar cortes para seleccionar la parte que sea de nuestro interés (Baker, 2016). Además de ello, el docente puede grabar su voz sobre el vídeo para añadir una introducción, explicar el contenido o incorporar subtítulos o comentarios. También, permite agregar preguntas, pausando el vídeo y evitar su avance hasta que sean respondidas por el alumno.

En Edpuzzle hay dos tipos de cuentas, para profesores y para alumnos. El contenido de cada clase es privado y puede ser visualizado sólo por el profesor y los alumnos que forman parte de la misma. Los alumnos tienen acceso a los vídeos, pueden verlos, pausarlos y rebobinarlos cuantas veces quieran. Además, permite realizar feedback por ambas partes. Es decir, el profesor puede comprobar las veces que los alumnos tuvieron que ver las diferentes partes del vídeo para hacer las tareas e identificarlas como las más difíciles de entender. Y por otro lado, el alumno puede recibirlo al responder las preguntas, ya que el formato permite al profesor incorporar la explicación respecto al motivo del acierto o error de las mismas. Para crear una clase en Edpuzzle es imprescindible programar la temporalidad durante la que deben realizarse las tareas por lo que si se establece antes de nuestra clase presencial, ya se estaría empleando FL.

En cuanto al ámbito de desarrollo de nuestro estudio, la Universidad Complutense de Madrid tiene, como centro de enseñanza superior, una plataforma de enseñanza telemática en la que se recogen materiales docentes elaborados por su profesorado y puesto a disposición de los alumnos en régimen de red privada (con exigencia de identificación y autentificación). Esta plataforma es Moodle 3.4 y en el Grado de Odontología su uso es apoyo fundamental para los docentes.

Moodle es una herramienta de software libre muy útil para la enseñanza. Su nombre proviene del acrónimo Modular Object Oriented Dynamic Learning Environment (Entorno Modular de Aprendizaje Dinámico Orientado a Objetos). Esta definición de Moodle quiere decir que permite crear "objetos de aprendizaje" con los que el profesor guía a los alumnos posibilitando su autoaprendizaje. Moodle se extiende exponencialmente por los centros de enseñanza de todo el mundo. Permite gestionar asignaturas así como otras utilidades, desde colgar contenidos multimedia (vídeos, imágenes, apuntes...), evaluar diferentes tareas del alumno, hasta realizar exámenes online. Es una herramienta ideal para gestionar la organización de las comunidades educativas y permitir trabajar y comunicarse a los distintos integrantes de las mismas. Como hemos señalado anteriormente, esta herramienta nos permite subir contenidos de nuestra asignatura antes de la clase expositiva tradicional por lo que es una herramienta útil para aplicar el FL (Ros, 2008). 
La incorporación de FL con vídeos en las prácticas preclínicas de Odontopediatría II (OP II), en $4^{\circ}$ curso del Grado de Odontología de la UCM, ya se llevó a cabo durante el pasado curso académico 2018/19 a través del Campus Virtual (Moodle) siendo muy bien acogido por los estudiantes. Los resultados académicos fueron buenos, no obstante, en la encuesta de satisfacción, el $85 \%$ de los alumnos contestó que utilizar otras plataformas más adecuadas para FL con respecto a la visualización de los vídeos les resultaría muy útil e interesante (Gallardo, 2019). Por todo ello, consideramos adecuado seguir utilizando FL en la asignatura, analizando si el uso de Edpuzzle mejora aún más el aprendizaje de los alumnos.

\section{Objetivos}

Tras la experiencia que nos proporcionó nuestro anterior estudio sobre la incorporación del aula invertida a los estudios de Grado en Odontología, decidimos desarrollar un nuevo trabajo durante este curso académico (2019/20). Los principales objetivos que nos planteamos fueron:

- Identificar, de forma objetiva, la adquisición de las destrezas que el alumno puede conseguir a través del FL en las prácticas preclínicas de OP II.

- Determinar si existen diferencias en cuanto al nivel de aprendizaje adquirido por los alumnos cuando empleamos Moodle o Edpuzzle como herramienta para el acceso al material educativo de las prácticas.

\section{Metodología}

El desarrollo de este estudio se llevó a cabo durante las prácticas preclínicas de tratamiento pulpar de la asignatura OP II del Grado de Odontología (UCM) y han participado 6 profesores ( 5 encargados de impartir las prácticas preclínicas y el responsable de la materia) y 74 alumnos matriculados en la asignatura.

La sistemática empleada para la aplicación de FL a las prácticas fue la siguiente:

- Todos los alumnos de $4^{\circ}$ curso matriculados en OP II que asistieron a la primera práctica (97 individuos) fueron informados del desarrollo de este estudio y realizaron un cuestionario de preselección. Como criterio de exclusión se determinó tener conocimientos previos sobre la materia, por lo que los alumnos repetidores o que 
hubieran realizado estudios sobre tratamientos pulpares en dentición temporal, fueron identificados a través de dicho cuestionario y se descartaron como participantes.

- El profesor responsable eligió 75 alumnos al azar (de los 92 seleccionados anteriormente) y los distribuyó aleatoriamente en 3 grupos: A, B y C.

- Se asignó cada grupo a una clase y 2 profesores, diferentes en cada grupo, se encargaron de explicar el posterior desarrollo de las prácticas.

- Todos los profesores participaron en la elaboración del material educativo demostrativo del procedimiento (vídeos) a realizar en las prácticas preclínicas (tratamientos pulpares en dientes artificiales montados en tipodonto).

- Todos los alumnos visualizaron los vídeos aunque, los grupos A y B tuvieron acceso ilimitado, 4 días antes de la primera práctica presencial, mientras que el grupo $\mathrm{C}$ los visualizó solamente una vez al inicio de la misma.

- Aunque todos los alumnos tenían acceso a la página de la asignatura en el Campus Virtual (Moodle), la visualización de los vídeos por esta vía se restringió al grupo A (Fig. 1).

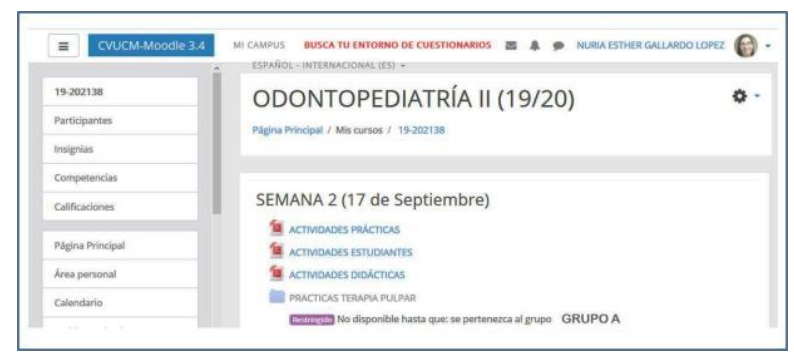

Fig. 1. Página de la asignatura Odontopediatría II en el Campus Virtual UCM.

- Edpuzzle fue la plataforma empleada con el grupo B. Los alumnos recibieron en su email UCM un enlace directo a la clase para poder ver los vídeos (Fig. 2).

Haciendo uso de las herramientas de esta plataforma, se incorporaron diversas preguntas a los vídeos que los alumnos debían contestar para ir avanzando en su visualización. Todas las preguntas aportaron a los alumnos feedback relacionado con las respuestas.

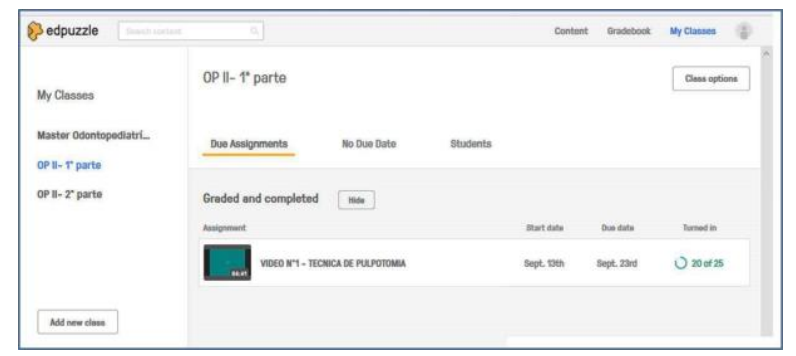

Fig. 2. Acceso a los vídeos en la Plataforma Edpuzzle. 
- Finalizadas las prácticas se solicitó a los alumnos la entrega de los dientes con los tratamientos realizados. Para garantizar el anonimato se entregó, a cada estudiante, una pequeña bolsa identificada con un número, en la que introdujeron sus trabajos.

- Se establecieron 4 indicadores de calificación para llevar a cabo una evaluación objetiva (Tabla 1). Un solo profesor realizó la calificación de todos los alumnos. En ningún momento tuvo conocimiento del grupo al que pertenecían las muestras. El rango de calificación final fue de 0 a 4 , siendo 4 la mejor calificación.

Tabla 1. Criterios de evaluación.

\begin{tabular}{lcc}
\hline \multicolumn{1}{c}{ Indicadores } & $\begin{array}{c}\text { Bien } \\
(\mathbf{1} \text { punto })\end{array}$ & $\begin{array}{c}\text { Mal } \\
\text { (0 puntos })\end{array}$ \\
\hline Eliminación del techo de la cámara pulpar & Completa & Incompleta \\
Debilidad de las paredes remanentes & No & Sí \\
Eliminación del tejido pulpar cameral & Completa & Incompleta \\
Profundidad de la cavidad > cámara pulpar & No & Sí \\
\hline
\end{tabular}

- Una vez realizada la evaluación, otro profesor repitió el procedimiento calificador en 30 dientes. Con ello se aseguró la concordancia interobservador.

- Se elaboró una tabla de Excel en la que se incorporaron los resultados de la evaluación y se llevó a cabo el análisis estadístico.

\section{Resultados}

De los alumnos que comenzaron el estudio, uno del grupo B no asistió a la última práctica y no entregó los dientes para su calificación, por lo que la muestra final disminuyó a 74.

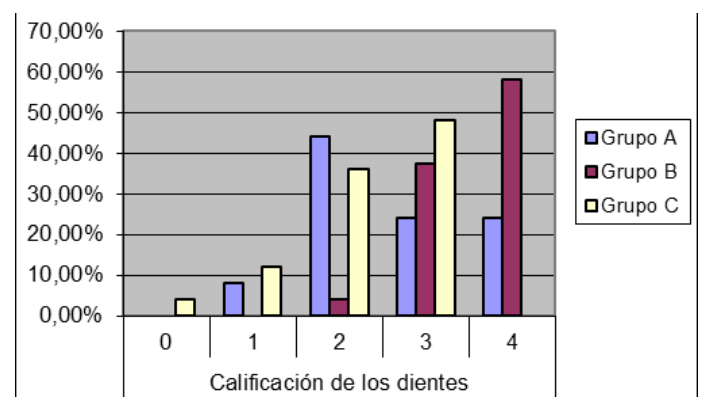

Fig. 3. Distribución de las calificaciones de los dientes. 
El grupo B, que empleó Edpuzzle, fue el que presentó mejores calificaciones frente a los otros dos, siendo la diferencia significativa entre los grupos ( $<<0.05$ ) (Fig. 3). El 58,3\% de los alumnos del grupo B obtuvieron la máxima calificación, pero en el grupo A solo lo hizo el 24\% y en el grupo C, no hubo ningún alumno que la alcanzara.

La tabla 2 presenta las calificaciones medias de cada grupo así como sus notas máximas y mínimas.

Tabla 2. Calificaciones distribuidas por grupos.

\begin{tabular}{ccccc}
\hline & Media & DE & Nota mínima & Nota máxima \\
\hline Grupo A & 2,64 & 0,95219 & 1 & 4 \\
Grupo B & 3,54 & 0,58823 & 2 & 4 \\
Grupo C & 2,28 & 0,84261 & 0 & 3 \\
\hline
\end{tabular}

Analizando cada indicador de evaluación, los mejores resultados se encontraron en "la eliminación del tejido pulpar" y de nuevo se produjo en el grupo B, ya que fue realizada correctamente por 23 de los 24 alumnos de dicho grupo.

La "eliminación del techo cameral" fue el indicador que peor elaboraron los alumnos en el conjunto total de la muestra. Solo 39 de los 74 alumnos hicieron una apertura completa de la cámara pulpar, pero sí es importante destacar que más de la mitad de los que lo hicieron bien (20 alumnos), pertenecían al grupo B.

En cuanto a los otros dos aspectos estudiados, "debilidad de paredes" y "profundidad", los valores estuvieron muy igualados, alcanzando buenas calificaciones (entre 20 y 22) en todos los grupos. Incluso, para este ítem el grupo A superó, aunque muy ligeramente, al grupo B ( $88 \%$ frente a $87,5 \%$ de dientes con tratamientos adecuados).

Cuando comprobamos el número de visualizaciones de los vídeos por alumno, éstas fueron muy superiores en el grupo B frente al A y debemos tener en cuenta que en el grupo C solo fue una y durante la práctica, lo cual puede relacionarse directamente con los resultados.

El éxito en el uso de la plataforma Edpuzzle frente a Moodle podría basarse, desde nuestro punto de vista, en la incorporación de preguntas a los vídeos que obligaron a los estudiantes a prestar más atención al contenido de los mismos. El feedback de las respuestas y la revisualización de fragmentos determinados de los vídeos dieron la posibilidad a los alumnos de comprobar, paso a paso, que habían adquirido los conocimientos necesarios para realizar los procedimientos y les ayudó en la posterior ejecución de las prácticas. 


\section{Conclusiones}

Este estudio indica que con la clase invertida se consiguen mejores resultados que con la clase magistral. Todos los alumnos que recibieron FL obtuvieron alguna puntuación en los trabajos realizados, en cambio, ninguno de la clase magistral obtuvo la máxima puntuación. Por tanto, consideramos que, a pesar de no encontrarse muy extendido en la docencia de Odontología, el FL es un método idóneo y recomendable para el Grado en Odontología.

Las calificaciones medias obtenidas por los trabajos preclínicos de los alumnos de OP II fueron 22,5\% superiores con Edpuzzle frente a Moodle. Esto nos lleva a concluir que Edpuzzle es una plataforma más adecuada para el desarrollo de FL. Su diseño y posibilidad de introducir preguntas multirrespuesta a lo largo de los vídeos aumenta el interés en los alumnos, y les estimula para hacer más visualizaciones, lo que proporciona una mejor comprensión de los conocimientos que se transmiten. Todo ello, repercute directamente en una mejora sustancial de su aprendizaje.

\section{Referencias}

Baker, A. (2016). Active Learning with interactive videos: Creating student-guided learning materials. Journal of Library \& Information Services in Distance Learning, 10(3-4), 79-87. Retrieved from http://doi.org/10.1080/1533290X.2016.1206776.

Bergmann, J. \& Sams, A. (2012). Flip your classroom: Talk to Every Class Every Day. Washington D.C: International Society for Technology in Education.

Bloom, B. S. (1971). Taxonomía de los Objetivos de la Evaluación. Buenos Aires, El Ateneo.

Campión, R. S. (2019). Conectando el modelo Flipped Learning y la teoría de las Inteligencias Múltiples a la luz de la taxonomía de Bloom. Magister: Revista miscelánea de investigación, 31(2), 45-54.

Gallardo, N.E., Caleya, A.M., Sánchez, E., Feijóo, G., Mourelle, R., de Nova, J. (2020). Incorporación del aula invertida en prácticas preclínicas de Odontopediatría. INNODOCT/19. Conferencia Internacional sobre Innovación, Documentación y Tecnologías Docentes. Editorial Universidad Politécnica de Valencia. Disponible en http://hdl.handle.net/10251/139715

Ros, I. (2008). Moodle, la plataforma para la enseñanza y organización escolar. Ikastorratza, eRevista de Didáctica 2. Disponible en http://www.ehu.es/ikastorratza/2_alea/moodle.pdf

Singh, V., Abdellahi, S., Maher, M. Lou, \& Latulipe, C. (2016). The video collaboratory as a learning environment. In Proceedings of the 47th ACM Technical Symposium on Computer Science Education (SIGCSE'16) (pp.352-357). Retrieved from http://doi.org/10.1145/2839509.2844588 